\title{
Competências colaborativas à formação do estudante: análise segundo domínios do Interprofessional Education Collaborative Expert Panel
}

\author{
Collaborative competences for student training: analysis \\ according to domains of the Interprofessional Education \\ Collaborative Expert Panel
}

\section{Wilton Nascimento Figueredo ${ }^{1}$ (1) Renata Meira Veras ${ }^{2}$ (1) Gilberto Tadeu Reis da Silva ${ }^{3}$ (1)}

\begin{abstract}
1Autor para correspondência. Universidade Estadual de Feira de Santana (Feira de Santana). Bahia, Brasil. wnfigueredo@uefs.br
\end{abstract} 2,3Universidade Federal da Bahia (Salvador). Bahia, Brasil. renata.veras@ufba.br, gilberto.tadeu@ufba.br

\begin{abstract}
RESUMO | OBJETIVO: Analisar, segundo os domínios do Interprofessional Education Collaborative Expert Panel, as competências colaborativas desenvolvidas pelo programa PermanecerSUS à formação dos estudantes de saúde. MÉTODO: Estudo qualitativo, sob a perspectiva da etnografia institucional, realizado em instituições de saúde públicas com estudantes, trabalhadores e usuários do sistema público de saúde. Os dados foram coletados por meio da observação participante e entrevista semiestruturada, e analisados com a análise de conteúdo temática, em consonância aos domínios do Interprofessional Education Collaborative Expert Panel. RESULTADOS: Participaram do estudo 60 pessoas, predominantemente do sexo feminino, divididas igualmente entre as unidades. Constatou-se que os indivíduos com menor idade e inseridos o mais precocemente em projetos interprofissionais, desenvolvem competências colaborativas com mais facilidade do que os trabalhadores dos serviços de saúde já atuantes dos serviços. CONCLUSÃO: O PermanecerSUS favorece aos estudantes ética/valores para a prática profissional, trabalho em equipe e corresponsabilidade. Entretanto, é preciso desenvolver entre os trabalhadores dos serviços de saúde a competência da comunicação interprofissional.
\end{abstract}

DESCRITORES: Relações interprofissionais. Comportamento cooperativo. Educação Superior. Educação Baseada em Competências. Desenvolvimento de pessoal.

\begin{abstract}
OBJECTIVE: To analyze, according to the Interprofessional Education Collaborative Expert Panel domains, the collaborative competencies developed by the Permanecersus program for the training of health students. METHOD: Qualitative study, from the perspective of institutional ethnography, conducted in public health institutions with students, workers, and users of the public health system. Data were collected through participant observation and semi-structured interviews and analyzed with thematic content analysis, in line with the domains of the Interprofessional Education Collaborative Expert Panel. RESULTS: 60 people participated in the study, predominantly female, equally divided among the units. It was found that individuals with younger age and inserted at an earlier stage in interprofessional projects develop collaborative skills more easily than health service workers already in service. CONCLUSION: The PermanecerSUS favors students ethics/values for professional practice, teamwork, and co-responsibility. However, it is necessary to develop the competence of interprofessional communication among health service workers.
\end{abstract}

DESCRIPTORS: Interprofessional relations. Cooperative behavior. Higher Education. Competency-based Education. Staff development. 


\section{Introdução}

A educação interprofissional (EIP) e as práticas colaborativas vêm sendo estimuladas pela Organização Mundial de Saúde e são tendências crescentes no cenário da formação do pessoal de saúde, em resposta ao paradigma predominante do ensino baseado na fragmentação e disciplinarização do conhecimento.

A EIP consiste no aprendizado entre duas ou mais profissões com a finalidade de promover e alcançar resultados promissores na área da saúde ${ }^{1}$. Já a prática colaborativa é a aplicação desse aprendizado nas mais diversas ações em benefício dos pacientes e suas famílias ${ }^{1}$.

Estudos recentes realizados em vários países desenvolvidos como Austrália² , Canadá3, Estados Unidos ${ }^{4-\underline{7}}$, Holanda ${ }^{8}$ e Japão 9 têm constatado que, apesar das discussões terem iniciado no século passado, a EIP é ainda pouco desenvolvida no ensino de graduação na área da saúde. Entretanto, quando estimulada, favorece a interprofissionalidade, promove aprendizado colaborativo e o desenvolvimento de competências capazes de incitar mudanças nos atuais modelos, paradigmas e estereótipos que pouco têm favorecido a centralidade do usuário no processo saúde-doença ${ }^{10-12}$.

No Brasil, o interesse pela EIP se difundiu principalmente logo após no início dos anos 2000. Desde então, destacam-se alguns estudos ${ }^{10,11}$ e experiências pontuais de projetos ${ }^{12}$ de diversas universidades com o objetivo de propiciar um currículo integrado aos estudantes dos cursos de Enfermagem, Fisioterapia, Farmácia, Medicina e Odontologia. Contudo, os resultados dessas pesquisas revelam barreiras para efetivar a EIP e, sobretudo, um ensino que ainda prioriza o desenvolvimento de competências técnicas (clínicas) em detrimento de competências colaborativas para a prática interprofissional.

Nessa perspectiva, em 2011, o grupo norte-americano Interprofessional Education Collaborative Expert Panel $(\text { IPEC })^{13}$, preocupado com a alta qualidade, segurança, acesso e cuidado centrado no paciente, definiu em quatro domínios, nos quais se inserem principais competências colaborativas para a prática interprofissional: valores/ética para prática interprofissional; funções e responsabilidades profissionais; comunicação interprofissional e equipes e trabalho em equipe. O domínio 1 do IPEC - valores/ética para a prática profissional - considera três diferentes abordagens fundamentais, respeito ético, cooperação multidisciplinar ética e relações profissionais conjuntas. O domínio 2 - funções/responsabilidades - fundamenta-se na compreensão dos papéis que cada profissional exerce para o cuidado integral, sendo um complementar ao outro. Já a comunicação interprofissional, que pertence ao domínio 3, é importante para melhorar a atenção integrada e em prol da escuta ativa. O objetivo do domínio 4 - equipes/trabalho em equipe - reside em promover um efetivo trabalho colaborativo entre os profissionais, para que realizem suas atividades como membros de um time ${ }^{13}$.

Nesse contexto, desde 2008, a Secretaria de Saúde do Estado da Bahia, Brasil, estimula a prática interprofissional e proporciona a vivência do trabalho colaborativo aos estudantes em formação por meio do programa PermanecerSUS.

O programa PermanecerSUS adotou como referência a Política Nacional de Humanização $\frac{14}{4}$ para alcançar o objetivo de proporcionar vivência aos estudantes da área da saúde, por meio da escuta ampliada, acolhimento, humanização, acionamento de redes internas e externas, em setores especializados e críticos de atendimento, como as emergências hospitalares, maternidade e centro de referência e diagnóstico do Sistema Único de Saúde (SUS).

Assim, em acordo à Organização Mundial de Saúde1 , que apontou a necessidade da implementação de políticas e programas direcionados à EIP, este estudo tem o objetivo de analisar, segundo os domínios do Interprofessional Education Collaborative Expert Panel, as competências colaborativas desenvolvidas pelo programa PermanecerSUS durante a formação dos estudantes de saúde.

\section{Método}

Estudo qualitativo sob a perspectiva metodológica da etnografia institucional. As pesquisas desenvolvidas sob o arcabouço da etnografia institucional apresentam uma abordagem sociológica ${ }^{15}$, pois se atentam às relações dominantes impostas e construídas nas organizações ${ }^{15,16}$. Sendo assim, permite realizar uma análise crítica acerca das relações de poder e das práticas normatizadas, preestabelecidas e burocráticas presentes nas instituições de saúde. 
Pesquisa realizada em quatro instituições públicas de saúde, em Salvador, região Nordeste do Brasil e receptoras do programa PermanecerSUS. As instituições foram selecionadas pelo tipo de serviço prestado à população, por apresentarem alta capacidade de atendimento diário e desenvolverem atividades institucionais heterogêneas: emergência de dois hospitais gerais, uma maternidade e um centro de referência de diagnóstico.

Os participantes da pesquisa foram estudantes (estagiários do programa PermanecerSUS), trabalhadores dos serviços de saúde (vinculados às unidades de saúde) e usuários do SUS (pessoas que utilizavam o serviço de saúde).

Os estudantes selecionados para o PermanecerSUS, atualmente, são procedentes de todos os cursos de saúde, Psicologia e Serviço Social, e de todas as instituições de ensino superior do Estado da Bahia. Quando em atividade, são divididos em grupos de cinco a seis membros de distintos cursos, a fim de concretizar uma experiência interprofissional e o fortalecimento do trabalho em equipe. Durante a prática há proibição de desempenho de procedimentos clínicos; contudo, existe o estímulo do fortalecimento da escuta, o direcionamento das demandas dos pacientes e a articulação de redes entre os serviços e os trabalhadores dos serviços de saúde.

Para a população dos estudantes, optou-se pela amostra do tipo não probabilística e selecionada com base no quantitativo máximo de cinco a seis estagiários alocados, conforme política de cada unidade/ instituição. Os usuários e trabalhadores dos serviços de saúde foram abordados aleatoriamente conforme seu interesse, disponibilidade para participar da pesquisa e pela saturação das informações colhidas no momento da entrevista. Para os estudantes, foram estabelecidos os seguintes critérios de inclusão: terem iniciado as atividades há pelo menos dois meses e estarem inseridos no atendimento à pessoa adulta, e para os trabalhadores dos serviços de saúde, definiu-se manter vínculo com a unidade há pelo menos seis meses, período considerado ideal pelos pesquisadores para conhecer as rotinas do atendimento e o programa PermanecerSUS. Foram excluídos os estudantes e trabalhadores dos serviços de saúde que durante a pesquisa estavam afastados ou de licença por qualquer motivo. Já os usuários foram selecionados com base nos seguintes critérios: deveriam ter sido atendidos por um dos estudantes e não terem expressado qualquer tipo de recusa em participar da pesquisa. Foram excluídos os usuários que tivessem agravamento do caso clínico e impossibilitados de falar. Os atendimentos realizados na pediatria foram excluídos por apresentar uma dinâmica diferenciada.

Nesse sentido, após aplicados os critérios de exclusão/inclusão e com vistas à homogeneização da amostra, formou-se grupos com cinco indivíduos de cada categoria, divididos equitativamente nas unidades, totalizando 60 participantes.

Como técnicas de pesquisa, optou-se pela observação participante, com auxílio do diário de campo, escrito diariamente, além de entrevistas semiestruturadas. Entretanto, neste artigo optou-se por trazer apenas os dados das entrevistas realizadas, ficando as informações da observação participantes discutidas em outro momento. Para as entrevistas, os participantes foram convidados pessoalmente e intencionalmente pelo pesquisador, mediante os critérios supracitados. Dessa forma, a pesquisa foi construída em dois momentos: no primeiro, realizada a observação participante nas quatro instituições de saúde, sendo reservado o período de um mês e 15 dias para a coleta de dados durante as manhãs e tardes, de segundas às sextas-feiras, no período de setembro de 2013 a janeiro de 2014. Os primeiros 15 dias foram exclusivos para observação, sem qualquer contato formal com os participantes do estudo. Entretanto, neste artigo os relatos.

O segundo momento da pesquisa, utilizada nos resultados e discussão deste artigo, aconteceu concomitantemente ao primeiro, mediante a realização das entrevistas semiestruturadas. Tais entrevistas possuíam um roteiro previamente construído pelos pesquisadores e dividido em dois blocos: aproximação e questões norteadoras; realizadas em ambiente exclusivo sem a interferência de outras pessoas, contínua e sem repetições.

O bloco de aproximação constituiu-se de perguntas basicamente iguais entre os usuários e trabalhadores dos serviços de saúde, tais como idade, sexo, profissão, tempo e motivo de internação e tempo de atuação na unidade, respectivamente. Os estudantes responderam a perguntas sobre o perfil sociodemográfico, econômico e universitário. 
As questões norteadoras para os usuários foram construídas para apreender suas percepções acerca do atendimento, a comunicação, orientação fornecida aos usuários, além do grau de satisfação e resolução do problema. Os trabalhadores dos serviços de saúde foram indagados sobre o entendimento do PermanecerSUS, a comunicação entre os membros da equipe e estudantes, as relações estabelecidas e a percepção sobre as mudanças no processo de trabalho após a inserção do programa na unidade. Os estagiários responderam sobre o trabalho em equipe, valores, ética, humanização, acolhimento, o desenvolvimento do seu trabalho com os usuários e a comunicação com os trabalhadores dos serviços de saúde da unidade.

Todas as entrevistas foram gravadas em aparelho formato MP3 e transcritas fidedignamente no programa de textos Microsoft Word® versão 2013, sob posse dos pesquisadores. As entrevistas com os estudantes, trabalhadores dos serviços de saúde e usuários duraram, em média, 30, 20 e 15 minutos, respectivamente.

Para a análise dos dados adotou-se a técnica de análise de conteúdo temática, com as etapas de pré-análise, investigação do material, análise dos resultados, interpretação e inferência ${ }^{17}$. As categorias de análise foram criadas à luz dos quatro domínios das competências propostos pelo IPEC $\frac{13}{}$.

Todos os participantes assinaram o Termo de Consentimento Livre e Esclarecido, em cumprimento aos preceitos éticos da Resolução $n^{\circ} 466 / 12$ do Conselho Nacional de Saúde, sendo a pesquisa aprovada pelo Comitê de Ética em Pesquisa do Instituto de Saúde Coletiva da Universidade Federal da Bahia sob protocolo $n^{\circ}$ 001-11/CEP-ISC.

Para preservar o anonimato das instituições e dos participantes, utilizou-se a codificação: unidade de saúde, seguido do participante mais numeração (1 a 5). Para as unidades de saúde, a sigla HG 1 (hospital geral 1), HG 2 (hospital geral 2), HM (hospital maternidade) ou CRD (centro de referência e diagnóstico); para os estudantes, a sigla EST, seguido do curso de graduação, por exemplo ENF (Enfermagem); a sigla PROF (trabalhadores dos serviços de saúde); e a sigla USU (usuários).

\section{Resultados}

Os resultados são apresentados em duas partes: na primeira, constam os achados referentes ao perfil dos participantes, e na segunda, a percepção dos entrevistados, categorizadas nos quatro domínios de competências do IPEC. Foram entrevistadas 60 pessoas, sendo 20 usuários, 20 estudantes e 20 trabalhadores dos serviços de saúde.

No grupo dos usuários, predominaram indivíduos do sexo feminino $(13 ; 65 \%)$, com média de idade de 36,6 anos, com ocupação predominante ao grupo S serviços pessoais e domésticos - da classificação nacional de atividade econômica (CNAE)18 do Instituto Brasileiro de Geografia e Estatística e média de tempo de internação igual a 7,3 dias. Os entrevistados do HG 2 não responderam ao tempo de internação.

O sexo feminino também predominou no grupo trabalhadores dos serviços de saúde, com 14 (75\%) das 20 entrevistadas. A média de idade foi de 44,5 anos e, em relação à profissão, houve diversidade: trabaIhadores da saúde - enfermeira, assistente social e técnica de enfermagem; trabalhadores administrativos - recepcionista e auxiliar administrativa; e trabaIhadores outros - seguranças e agentes de portaria. O tempo médio de atuação profissional nas quatro unidades foi de 12,4 anos.

Para o grupo dos estudantes, houve o maior percentual do sexo feminino, com 16 (85\%) das 20 entrevistadas, média de idade igual a 22,7 anos, predominância dos cursos de Bacharelado Interdisciplinar em Saúde, Enfermagem e Medicina, em detrimento de Odontologia, Saúde Coletiva e Serviço Social, que contaram com apenas um entrevistado em todas as unidades. O tempo médio de realização do curso de graduação foi de 6,2 período/ semestres.

A segunda parte dos resultados, percepção dos entrevistados, foi dividida conforme os quatros domínios do IPEC.

No domínio 1 IPEC - Valores/Ética para a Prática Interprofissional - os entrevistados expressaram reconhecer a importância do PermanecerSUS para melhorar o atendimento nas unidades onde era desenvolvido. Destacaram-se, sobretudo, a atuação acolhedora e humanizada dos estagiários que participavam do referido programa: 
O PermanecerSUS ajuda na ouvidoria também, por exemplo, aqui tivemos um caso na ouvidoria que foi resolvido pelo atendimento ético do estagiário e sua capacidade de conduzir e respeitar, mesmo sem saber, o caso da usuária. (HM - PROF.2)

A presença deles (sobre os estagiários) acaba interferindo, ajudando, o atendimento dos usuários, pois eles têm aquele jeito de ouvir correto, porque os usuários daqui precisam muito, e os meninos vêm da faculdade bem diferentes, bem acolhedores [...] Era isso que faltava (sobre o acolhimento). As pessoas, os usuários, se sentem mais confiantes [...] É você escutar, compreender, perguntar, ouvir, compreender [...] Então isso ajudava na solução do problema do paciente. (CRD - PROF.5)

$O$ atendimento que mais gostei foi dele (sobre o estagiário). Ele só não me encaminhou, mas me ouviu, porque muitos nem querem saber mais, só do problema e pronto? (CRD - USU.3)

No segundo domínio IPEC - Funções/ Responsabilidades, amparados na compreensão dos papéis que cada profissional exerce para o cuidado integral, os participantes ressaltaram as mudanças e os benefícios proporcionados pelo PermanecersUS aos estudantes no que se refere à oferta de cuidado fundamentado em um novo paradigma de saúde:

O PermanecerSUS me proporciona aproximação com a realidade, a gente estuda as leis, a questão dos direitos, a história da saúde pública, a gente vivencia

[...] Nos fornece elementos de realidade, de sair da universidade, pensar como funcionários e saber o dever de cada um. (HG 1 - EST.SSO.4)

O PermanecerSUS me ajuda a compreender o que cada profissional faz, isso vai me ajudar a ter uma visão de como é a profissão de cada um para melhor benefício do paciente. (HG 2 - EST.BIS.4)

Hoje eu entendo, mas antes não, a função do estagiário do PermanecerSUS [...] Começou assim, a interação deles com os usuários [...] se aproximando da gente daqui da unidade com os pacientes. (CRD - PROF.5)

Com o terceiro domínio IPEC - Comunicação Interprofissional - apreende-se o prejuízo causado pela falta de comunicação entre os diversos membros da equipe de saúde, conforme é possível observar nas falas a seguir:
Estamos sempre nos comunicando com a equipe de enfermagem, com a assistente social [...] A principal dificuldade em desenvolver o nosso trabalho é a comunicação de quem está aqui para atender o paciente [...] A comunicação é um tabu aqui dentro [...] O paciente já está tão saturado, porque já peregrinou em outras unidades e não teve atendimento.

(HG 1 - EST.SSO.4)

Você me faz uma pergunta difícil. Sobre comunicação? Não há não (silêncio e respira profundamente) é tudo muito individualizado. O profissional fica ali na sua área e pronto. Não há uma reunião, não há entrosamento. (HG 1 - PROF.5)

Foi bem duro a comunicação com os profissionais, porque eram poucos que paravam para conversar e ouvir a gente. A gente tentava resolver o problema do paciente, mas era difícil que eles (profissionais) nos ouvissem. (HM - EST.FIS.4)

No domínio 4 IPEC - Equipes e trabalho em equipe - os participantes mencionaram a importância de realizarem um efetivo trabalho em equipe, pois isso proporciona benefícios prontamente percebidos por todos os envolvidos, inclusive pacientes:

O PermanecersUS me proporciona o trabalho em equipe [...] E também a viver com as dificuldades [...] com problemas que poderiam serem resolvidos se fossem discutidos entre os membros da equipe. (HG 2 - EST.BIS.4)

Nosso programa dialoga com as outras pessoas sim, pois a partir do momento que não temos competência em resolver uma demanda, vamos atrás dos responsáveis, como chefe de plantão, assistente social.

(CRD - EST. FON.3)

A gente ia atrás do profissional, tentava solucionar e agilizar o caso do usuário [...] No início foi ruim, porque muitos pensavam que estávamos lá para fiscalizar, mas no final alguns até chegavam na gente para resolvermos e irmos até o paciente, tentar fazer essa rede [...] Acho que alguns entenderam qual era nosso papel (HM - EST. BIS.1).

Ele me ouviu e aí pediu para eu esperar. Depois voltou e foi comigo na sala de outro profissional e conversamos, os três juntos. Foi ótimo, porque se entenderam. Parece que já até sabiam como era o meu caso (CRD - USU.1). 


\section{Discussão}

Ao analisar a percepção dos usuários, trabalhadores e estudantes, os resultados deste estudo evidenciam a tendência da formação em saúde na contemporaneidade: pautada na interprofissionalidade, centrada nos pacientes e suas famílias ${ }^{13}$, direcionada à qualidade ${ }^{19}$ dos serviços prestados e sobretudo dialogada entre os diferentes atores que compõem o sistema de saúde.

A predominância do sexo feminino nas três categorias entrevistadas acompanha os dados estatísticos brasileiro: as mulheres procuram mais os serviços de saúde, possuem maior qualificação profissional, dedicam seu tempo aos cuidados de pessoas e/ou afazeres domésticos, contudo, em relação aos homens, ocupam menos da metade dos cargos gerenciais ${ }^{20}$. Neste sentido, sugere-se da importância que as estudantes do programa PermanecerSUS desempenham para a efetivação da educação interprofissional, pois pessoas do sexo feminino, conforme pesquisa ${ }^{21}$, demonstram maior aceitabilidade e interesse em desenvolver as competências colaborativas do que o sexo masculino.

Em relação ao tempo/período da graduação e à idade dos estagiários entrevistados nesta pesquisa, são reconhecidas as potencialidades da inserção precoce desse grupo em projetos que associem educação e trabalho com as realidades do sistema de saúde. Entre essas potencialidades estão o aumento da capacidade de aprendizado colaborativo, fortalecimento do trabalho em equipe e de melhor comunicação entre os pares. Tal constatação também é discutida em dois estudos, um brasileiro 21 e outro asiático ${ }^{22}$, que demonstraram significância estatística para a idade e período do curso de graduação: quanto mais jovem, maior a disponibilidade para o aprendizado colaborativo, em compreender suas funções/responsabilidades em equipe e promover uma prática colaborativa e ética nos serviços de saúde.

Acerca dos domínios do IPEC, reconhece-se que os valores apreendidos ao longo da formação e da experiência de cada um para o desenvolvimento de práticas colaborativas. As falas dos profissionais destacam as atitudes de acolhimento pautadas na ética dos estudantes do PermanecerSUS. Os trabalhadores dos serviços de saúde de forma geral reconhecem que a prática em saúde demanda um trabalho que transcende os afazeres individualizados de cada profissão, indo além do que pode ser apreendido na formação ${ }^{23}$. Valorizar esses princípios éticos possibilita a cooperação para o exercício de práticas transformadoras ${ }^{11}$.

O segundo domínio do IPEC, relativo aos papéis e responsabilidades, relata da pertinência da construção de competências variadas no trabalho em equipe, quanto à formação profissional como backgrounds culturais ${ }^{13}$. Ademais, as responsabilidades assumidas interprofissionalmente precisam de exatidão por todos os membros da equipe, a fim de conhecer suas funções, como compreender a dos demais membros. Nesse sentido, os entrevistados apontam os efeitos promissores do PermanecerSUS na construção dessa competência e na compreensão, individual e coletiva, do seu papel na condução do trabalho em saúde.

As falas de alguns entrevistados denotam a importância da interdisciplinaridade na formação, pré-requisito para a atuação interprofissional, baseada no trabalho em equipe, discussão de papéis profissionais, compromisso na solução de problemas e negociação na tomada de decisão ${ }^{23,24}$.

Por outro lado, entrevistados relatam as dificuldades em desenvolver as práticas colaborativas entre os trabalhadores de saúde. Analisando o perfil dos trabalhadores dos serviços de saúde já atuantes das unidades: média de idade $(44,5)$ e tempo de atuação (12,4 anos), pode-se constatar que seu modo de trabalho pode estar relacionado com o tipo de formação que tiveram. Tal evidência é também demonstrada em estudos ${ }^{21-22}$, que comprovam a relação direta entre o modelo formativo e as práticas desenvolvidas em saúde. Ademais, visto que a discussão sobre EIP emergiu com intensidade por volta dos anos 2010', podemos inferir que os trabalhadores dos serviços de saúde com mais tempo de atuação vivenciaram uma formação pautada em currículos não integrados e na disciplinarização do conhecimento, consequentemente, podem apresentar distanciamento do terceiro e quarto domínio IPEC.

A comunicação interprofissional, é crucial para o desenvolvimento de competências colaborativas e do trabalho em equipe ${ }^{13}$; no entanto, exige dos profissionais o abandono de trabalhos individualizados e não dialógicos, e que, infelizmente, estão circunscritas à sua prática, conforme nos apontam os discursos dos entrevistados. 
Nesse sentido, o conhecimento e a compreensão da dinâmica do grupo são essenciais para o desenvolvimento do trabalho colaborativo, caso contrário pode ocorrer dificuldade no processo, como evidencia alguns entrevistados ao relatarem o incômodo dos profissionais diante dos estagiários do PermenecerSUS. Entretanto, a atitude dos estudantes do PermanecerSUS em estabelecer vínculos comunicativos entre os atores supera as barreiras e desenvolve práticas colaborativas. Assim, a efetivação do trabalho em equipe, centrada no paciente, aumenta o grau de satisfação do usuário como pontuado pelo participante.

A proposta do PermanecersUS é propiciar a comunicação, conhecimento e entrosamento dos estudantes com as particularidades de outras profissões da área da saúde, culminando um trabalho em equipe nas instituições receptoras do programa ${ }^{24}$. No entanto, destacamos a necessidade de incentivar a participação de outras áreas da saúde, diferentes da Enfermagem e Medicina, majoritariamente presentes e hierárquicas nas unidades de saúde. Essa evidência não é só peculiar ao Brasil, pois estudo multicêntri$\mathrm{CO}^{7}$, norte-americano, sobre as competências colaborativas, mostrou a predominância dos referidos cursos, em detrimento de outros, como a Odontologia. Assim, relações profissionais hierarquizadas tendem a diminuir a prática colaborativa entre os demais trabalhadores de saúde ${ }^{25}$.

Foram limitações do estudo o projeto inicial sobre programa PermanecerSUS estar direcionado ao referencial teórico da Política Nacional de Humanização e não exclusivamente às práticas colaborativas, não ter incorporado outros atores às entrevistas, a escolha do modelo Interprofessional Education Collaborative Expert Panel e a regionalização do estudo. A escolha do referido modelo pode ser uma limitação, uma vez que existem outros construídos mundialmente e que também podem contribuir para a EIP. Entretanto, os dados podem ser extensíveis e aplicados em outras regiões, desde que consideradas as suas particularidades.

\section{Conclusão}

As competências colaborativas proporcionadas pelo programa PermanecerSUS aos estudantes são: ética/ valores para a prática profissional, trabalho em equipe e corresponsabilidade. Entretanto, a competência comunicação interprofissional se apresentou em menor adesão entre os trabalhadores dos serviços de saúde.

\section{Agradecimentos}

A referida pesquisa foi financiada pela Fundação de Amparo à Pesquisa do Estado da Bahia (Fapesb), termo de outorga PPP 057/2011.

\section{Contribuições dos autores}

Figueredo WN colaborou na concepção, delineamento da pesquisa, coleta dos dados, redação, análise, revisão crítica do artigo e aprovação final da versão a ser publicada. Veras RM e Silva GTR colaboraram no delineamento da pesquisa, na redação, análise, interpretação dos dados, revisão crítica do artigo e aprovação final da versão a ser publicada.

\section{Conflitos de interesses}

Nenhum conflito financeiro, legal ou político envolvendo terceiros (governo, empresas e fundações privadas, etc.) foi declarado para nenhum aspecto do trabalho submetido (incluindo, mas não se limitando a subvenções e financiamentos, participação em conselho consultivo, desenho de estudo, preparação de manuscrito, análise estatística, etc.).

\section{Referências}

\begin{abstract}
1. Health Professions Network, Nursing \& Midwifery, Human Resources for Health. Framework for action on interprofessional education \& collaborative practice [Internet]. Geneva: World Health Organization; 2010. Disponível em: http://whqlibdoc.who. int/hq/2010/WHO_HRH_HPN_10.3_eng.pdf
\end{abstract}

2. Wilson AJ, Palmer L, Levett-Jones T, Gilligan C, Outram S. Interprofessional collaborative practice for medication safety: Nursing, pharmacy, and medical graduates experiences and perspectives. J Interprof Care. 2016;30(5):649-54. https://doi.org/1 $\underline{0.1080 / 13561820.2016 .1191450}$

3. Goldman J, Kitto S, Reeves S. Examining the implementation of collaborative competencies in a critical care setting: Key challenges for enacting competency-based education. J Interprof Care. 2017;1-9. https://doi.org/10.1080/13561820.2017.1401987

4. Hermann CP, Head BA, Black K, Singleton K. Preparing Nursing Students for Interprofessional Practice: The Interdisciplinary Curriculum for Oncology Palliative Care Education. J Prof Nurs. 2016;32(1):62-71. https://doi.org/10.1016/j.profnurs.2015.06.001 
5. Peeters MJ, Sexton M, Metz AE, Hasbrouck CS. A teambased interprofessional education course for first-year health professions students. Curr Pharm Teach Learn. 2017;9(6):1099110. https://doi.org/10.1016/j.cptl.2017.07.006

6. Dow AW, Diazgranados D, Mazmanian PE, Retchin SM. An exploratory study of an assessment tool derived from the competencies of the interprofessional education collaborative. J Interprof Care. 2014;28(4):299-304. https://doi.org/10.3109/13561 $\underline{820.2014 .891573}$

7. Lockeman KS, Dow AW, DiazGranados D, McNeilly DP, Nickol $D$, Koehn ML, et al. Refinement of the IPEC Competency SelfAssessment survey: Results from a multi-institutional study. J Interprof Care. 2016;30(6):726-31. https://doi.org/10.1080/135618 $\underline{20.2016 .1220928}$

8. Vries-Erich J, Reuchlin K, Maaijer P, van de Ridder JMM. Identifying facilitators and barriers for implementation of interprofessional education: Perspectives from medical educators in the Netherlands. J Interprof Care. 2017;31(2):170-4. https://doi. org/10.1080/13561820.2016.1261099

9. Haruta J, Sakai I, Otsuka M, Yoshimoto H, Yoshida K, Goto M, et al. Development of an interprofessional competency framework in Japan. J Interprof Care. 2016;30(5):675-7. https://doi.org/10.108 0/13561820.2018.1426559

10. Silva JAM, Peduzzi M, Orchard C, Leonello VM. Educação interprofissional e prática colaborativa na Atenção Primária à Saúde. Rev Esc Enferm USP. 2015;49(esp2):16-24. https://doi. org/10.1590/S0080-623420150000800003

11. Souza GC, Peduzzi M, Silva JAM, Carvalho BG. Trabalho em equipe de enfermagem: circunscrito à profissão ou colaboração interprofissional?. Rev Esc Enferm USP. 2016;50(4):642-9. https:// doi.org/10.1590/S0080-623420160000500015

12. Aguilar-da-Silva RH, Scapin LT, Batista NA. Avaliação da formação interprofissional no ensino superior em saúde: aspectos da colaboração e do trabalho em equipe. Avaliação (Campinas). 2011;16(1):165-84. https://doi.org/10.1590/S141440772011000100009

13. Interprofessional Education Collaborative Expert Panel. Core competencies for interprofessional practice: Report on an expert panel [Internet]. Washington, D.C: Interprofessional Education Collaborative; 2011. Disponível em: https://www.aacom.org/docs/ default-source/insideome/ccrpt05-10-11.pdf?sfvrsn=77937f97 2

14. Ministério da Saúde (Brasil), Secretaria de Atenção à Saúde, Núcleo Técnico da Política Nacional de Humanização. HumanizaSUS: Documento base para gestores e trabalhadores do SUS [Internet]. Brasília: Ministério da Saúde; 2010. Disponível em: http://bvsms.saude.gov.br/bvs/publicacoes/humanizasus documento gestores trabalhadores_sus.pdf
15. Harvey $C L$, Baret $C$, Rochefort CM, Meyer A, Ausserhofer $D$, Ciutene R, et al. Discursive practice - lean thinking, nurses' responsibilities and the cost to care. J Health Organ Manag. 2018;32(6):762-78. https://doi.org/10.1108/jhom-12-2017-0316

16. Ross CA, Jakubec SL, Berry NS, Smye V. "A Two Glass of Wine Shift": Dominant Discourses and the Social Organization of Nurses' Substance Use. Glob Qual Nurs Res. 2018;5:1-12. https:// doi.org/10.1177/2333393618810655

17. Fornari LF, Sakata-So KN, Egry EY, Fonseca RMGS. As perspectivas de gênero e geração nas narrativas de mulheres abusadas sexualmente na infância. Rev. Latino-Am. Enfermagem. 2018;26:e3078. https://doi.org/10.1590/1518-8345.2771.3078

18. Comissão Nacional de Classificação. Classificação Nacional de Atividades Econômicas - CNAE [Internet]. Instituto Brasileiro de Geografia e Estatística; 2018. Disponível em: https://cnae.ibge.gov. br/classificacoes/por-tema/atividades-economicas

19. Posey S, Brady J, Pate K, Smith J, Peveto S. Description and assessment of a pilot interprofessional education case competition activity. Curr Pharm Teach Learn. 2018;10(4):505-10. https://doi.org/10.1016/j.cptl.2017.12.009

20. Instituto Brasileiro de Geografia e Estatística. Estatísticas de gênero: indicadores sociais das mulheres no Brasil [Internet]. Estudos e pesquisas: informação demográfica e socioeconômica. 2018;38:1-12. Disponível em: https://biblioteca.ibge.gov.br/ visualizacao/livros/liv101551_informativo.pdf

21. Nuto SAS, Lima Júnior FCM, Camara AMCS, Gonçalves CBC. Avaliação da Disponibilidade para Aprendizagem Interprofissional de Estudantes de Ciências da Saúde. Rev Bras Educ Med. 2017;41(1):50-7. https://doi.org/10.1590/198152712015v41n1rb20160018

22. Aziz Z, Teck LC, Yen PY. The attitudes of medical, nursing and pharmacy students to inter-professional learning. Procedia - Soc Behav Sci. 2011;29:639-45. https://doi.org/10.1016/j. sbspro.2011.11.287

23. Figueredo WN, Rios DRS, Teles PSF, Magalhães TKS, Véras RM. Permanecer SUS: a (re) formação em saúde sob a perspectiva do acolhimento e da humanização. Rev Enferm UFSM. 2015;5(3):46575. https://doi.org/10.5902/2179769213593

24. Figueredo WN, Veras RM, Silva GTR, Cardoso GMP. Práticas colaborativas nas urgências em Saúde: a interprofissionalidade do Programa PermanecerSUS, Secretaria Estadual de Saúde da Bahia, Brasil. Interface (Botucatu). 2018;22(supl.2):1697-704. https://doi.org/10.1590/1807-57622017.0678

25. Freire Filho JR, Costa MV, Magnago C, Forster AC. Atitudes para a colaboração interprofissional de equipes da Atenção Primária participantes do Programa Mais Médicos. Rev. LatinoAm. Enfermagem. 2018;26:e3018. https://doi.org/10.1590/1518$\underline{8345.2731 .3018}$ 